\title{
EFFECTIVENESS AND SAFETY OF A THERMAL INSULATING COVERAGE ON THE TOP OF THE CRYO- CABIN DURING A PARTIAL-BODY CRYOSTIMULATION
}

Massimo De Nardi ${ }^{1,2}$, Sara Silvani ${ }^{1}$, Carlo Facheris ${ }^{1}$, Martina Pagnoncelli ${ }^{1}$, Ambra Bisio $^{2,3}$, Emanuela Faelli ${ }^{2,3}$, Antonio La Torre ${ }^{4,5}$, Piero Ruggeri ${ }^{2,3}$, Roberto Codella ${ }^{4,6}$

1. Krioplanet Ltd, Treviglio, Bergamo, Italy

2. Department of Experimental Medicine, Università degli Studi di Genova, Genoa, Italy

3. Centro Polifunzionale di Scienze Motorie, Università degli Studi di Genova, Genoa, Italy

4. Department of Biomedical Sciences for Health, Università degli Studi di Milano, Milan, Italy

5. IRCCS Istituto Ortopedico Galeazzi, Milan, Italy

6. Department of Endocrinology, Nutrition and Metabolic Diseases, IRCCS MultiMedica, Milan, Italy

\section{Corresponding Author:}

Roberto Codella

Department of Biomedical Sciences for Health

Università degli Studi di Milano

Via F.1li Cervi 93, 20090 Segrate (Milano) - Italy

Phone: $\quad+390250330356$

Fax: $\quad+390250315152$

E-mail: $\quad \underline{\text { roberto.codella@unimi.it }}$ 


\begin{abstract}
Partial Body Cryostimulation (PBC) consists of exposing minimally dressed participants to very cold air, in a specially designed cabin (cryo-cabin), for a short period of time. In recent years, cryo-cabins have been launched with a coverage to limit thermo-dispersion, however a validation study is lacking. The aim of this study was to compare thermal responses after a PBC protocol in an open cryo-cabin or into a cryo-cabin closed at the top with a polyurethane-made lid. Eighteen young male adults completed the two $150 \mathrm{~s}$ PBC sessions in a cross-over fashion. Temperature of the inner cabin and thermal responses were measured prior and 1, 5, 10, 20 min after completing each PBC session. When covered, cryo-cabins maintained a lower temperature in the front with respect to the back. There was a significant interaction regarding coverage*time* position with a main effect of coverage. Body surfaces were significantly colder under covered condition compared to open PBC. Subjects perceived a greater thermal discomfort during a covered PBC rather than during an open condition. For the first time, the use of a coverage placed on the top of the cabin was demonstrated to maintain lower temperatures of the inner environment and the users' skin with respect to a standard PBC. This evidence indicates that practitioners and clinicians making use of PBC can successfully and safely cover cryo-cabins with an insulating lid so to improve the effectiveness of their treatments.
\end{abstract}

Key words: cryotherapy, skin temperature, thermal imaging, thermal discomfort. 


\section{INTRODUCTION}

Partial-body cryostimulation (PBC) consists of exposing minimally dressed participants to very cold air, in a specially designed cabin (cryo-cabin), for a short period of time. PBC treatment is usually performed in a cryo-cabin called cryo-sauna. It is an open tank in which the subject is exposed to temperatures below $-130^{\circ} \mathrm{C}$, excluding the head and neck. According to (Bouzigon et al., 2016) cryo-sauna was the first technology for cooling the entire body: it was introduced by Japanese Professor Toshiro Yamauchi in the late seventies (YAMAUCHI and T., 1986). Then it was developed in the Eastern Europe between 1980 and 1990 (Zagrobelny et al., 1992). PBC differs from whole-body cryostimulation (WBC) for various

reasons. In PBC, one single user is exposed to very-low temperatures. In WBC, one to several subjects could perform the cryo-treatment simultaneously in the same chamber wholly exposed to the cold air. According to Bouzigon et al. (Bouzigon et al., 2016), the temperatures registered between and within different cooling methodologies may vary depending on protocols. During a cryo-session, while the temperature at the outlet of the nozzle is known in real time, the actual temperature inside the cabin or the chamber is hard to be determined accurately, and this was done only in two studies (Bouzigon et al., 2014; Savic et al., 2013). As explained also by Bouzigon et al. (Bouzigon et al., 2017), the efficiency of the cryoexposure is traditionally reported by measuring the variation of the skin temperature registered throughout the cryo-session. Savic and colleagues (Savic et al., 2013) assessed the variation of the skin temperature with a thermal camera, obtaining thermo-images of the entire human body surface (Matos et al., 2015).

Practitioners are uncertain when it comes to recommendations on a standardized protocol, in terms of duration and operating temperature, because each cryo-device is different from another and manufacturers do not provide objective data regarding the temperatures inside the 
cabins (Bouzigon et al., 2016). Nowadays, the market of the very-low temperature exposure has considerably grown worldwide, and, at present, there are many cryo-cabin and cryochamber manufactures around the world. Despite this exponential growth of equipment and methodologies, the employed protocols are purely arbitrary and rarely meet scientific criteria (Bouzigon et al., 2016). Recently, as regards the cryo-cabin, an increasingly numbers of practitioners are introducing a variant in the standard $\mathrm{PBC}$ procedure, that is covering the top of the cabin with a polyurethane layer, with a hole in the middle, so that the head is outside the cabin, whereas the shoulder area is fully exposed to the cold air. Inside the cryo-cabin, in fact, the cryogenic ambient is regulated by continuous injections of nitrogen every time the inner temperature falls below a set value (e.g. $-140^{\circ} \mathrm{C}$ ). Therefore, the inner temperature is not constant for three reasons at least: 1) the user's body is a continuous source of warm temperature; 2) the injected nitrogen, being heavier than atmosphere air, falls downward and fluctuations are paralleled by the rate of nitrogen injections; 3 ) at the top of the open tank, the cryogenic ambient is not isolated, hence the cooled air is warmed by the room air. This procedure should aid to insulate the cold ambient inside of the cabin from the surrounding temperature, contributing to the lowering of skin temperature in the upper body regions. To the authors' knowledge, there are no studies published on the effects of a covered cryocabin on skin temperature after a single PBC treatment. Therefore, the aim of this study is to examine thermal response after a standardized protocol of PBC (Fonda et al., 2014) in an open cryo-cabin, and compare it to that one following a control cryo-session using a coverage with a polyurethane-made lid. Our hypothesis is that skin temperature of the upper body would be lower after cryo-exposures with the coverage. 


\section{METHODS}

\subsection{PARTICIPANTS}

Based on the a priori sample-sized determination (software package, $G *$ Power 3.1.9.2), with a statistical power of 0.8 , a probability level of 0.05 , an effect size $f$ of 0.5 (which corresponds to a $\left.\eta_{p}^{2}=0.107\right)$, twelve subjects would have been sufficient to assess the majority of the sought effects. However, to face a possible drop-out of one third of the subjects, eighteen healthy young male adults were enrolled for this study. The characteristics (mean \pm SD) of the subjects were: age, $23.6 \pm 3.8$ years; height $1.78 \pm 0.05 \mathrm{~m}$; body weight $71.13 \pm 6.2 \mathrm{~kg}$ and BMI $22.35 \pm 1.2 \mathrm{~kg} / \mathrm{m}^{2}$. First, the subjects were examined by a physician to exclude any contraindication to $\mathrm{PBC}$ and gave their written informed consent to participate. They were also informed that they have the right to withdraw, at any stage of the study, without giving reasons. The study was approved by the Ethics Committee of the University of Milan and all participants signed a statement of informed consent at enrollment. Participants were requested not to smoke, drink alcohol or hot drinks for 24 hours before the experiment.

\subsection{STUDY DESIGN}

This investigation was designed as a randomized-crossover trial. The order of the two experimental conditions (with and without coverage) was randomly allocated based on balanced permutations generated by a computer program.

The protocol envisaged two consecutive sessions of cryostimulation at the same hour of the day (from 16:00 to 18:00). In one day, a cryostimulation was performed under standard conditions (without polyurethane layer), while in another day subjects were tested with a covered PBC. In this latter case, a thermal 5-cm-thick insulating coverage was placed on the top of the cabin, with a hole in the middle in a way that the subject could perform the session with his head out of the cabin. Amid the hole created in the middle of the coverage and the 
neck of each participants was placed a sanitized towel, in order to prevent gas escaping from the coverage. Each subject arrived at the laboratory 30 min before $\mathrm{PBC}$, where they rest in a sitting position wearing only shorts, woolen socks and wooden clogs to acclimate to the room temperature $\left(20.0 \pm 0.5^{\circ} \mathrm{C}\right)$.

Before the exposure, thermal imaging of the whole body from front and back was performed (Fig. 1) by using a ThermoVision SC640 thermal imaging camera (Flir Systems, Danderyd, Sweden) in accordance with the standard protocol of infrared imaging in medicine (Costello et al., 2012; Ring and Ammer, 2015, 2012). Thermal imaging was repeated immediately after the PBC session, and after 5, 10, 20 minutes. Throughout the measuring of the skin temperature, participants sat on a bench in a controlled temperature room $\left(20.0 \pm 0.5^{\circ} \mathrm{C}\right)$. Subjects were instructed not to talk, eat, drink, or do anything that could influence the thermal response. The camera, with the emissivity set in the range of 0.97-0.98, was mounted on a tripod and positioned in a way to focus on the entire body of the subjects. The distance between the camera and the subjects was kept constant at $3 \mathrm{~m}$. The mean temperature of sixteen ( 8 front, 8 back) body regions of interest (ROIs) was calculated by averaging the skin temperatures with commercially available software (Thermacam Researcher Pro 2.10, version 5.13.18031.2002, Flir Systems 2015, Danderyd, Sweden). The sixteen ROIs analyzed were front \& back legs, thighs, trunk, chest, forearms.

During a cryosession, each participant was exposed to very-low temperatures, according to literature (Fonda et al., 2014) for $150 \mathrm{~s}$ cryo-cabin at a temperature raging from -130 to -170 ${ }^{\circ} \mathrm{C}$ (Cryomed Pro, Criomed, Ltd, Kherson, Ukraine). Three-second-long injections of liquid nitrogen were done every time the temperature in the cabin rose above $-130^{\circ} \mathrm{C}$. According to the manufacturer's recommendations, each subject wore shorts, a pair of gloves, woolen sock and wooden clogs to prevent the occurrence of frostbites. Participants were assisted by an expert machine operator to walk on the same spot continuously for all the time of the 
treatment. On every session, participants were asked to rate their thermal discomfort on a 10$\mathrm{cm}$ visual-analogue scale, where $0=$ "not feeling cold in any way" and $10=$ "feeling unbearably cold". Thermal discomfort was assessed every $15 \mathrm{~s}$, starting $30 \mathrm{~s}$ after the beginning of the session (Fonda et al., 2014; Leon et al., 2008).

\subsection{MEASUREMENT OF THE INNER CABIN TEMPERATURE}

One thermocouple thermometer with T probe (HD2178.2, Delta Ohm Srl, Padova, Italy) was used to measure the temperature inside the cryo-cabin. The thermocouple was placed nearby the middle of the tibia, evenly placed close to the front or the rear right side of the subject (Fig. 2), according to the randomization procedure (§ 2.5). A trained operator compared the inner temperature of the cabin with that one shown at the thermometer display every $15 \mathrm{~s}$ throughout the entire duration of the cryo-session.

\subsection{FABRICATION OF THE THERMAL INSULATING COVERAGE}

A rigid polyurethane foam was adopted to the purpose (Fig. 3). The choice of this cryogenic insulation material was based on the fact that rigid polyurethane foams perform well in most areas of low-temperature insulations for its low thermal conductivity (Stirna et al., 2011). In fact, polyurethane products with density ranging from 30 to $200 \mathrm{~kg} \mathrm{~m}^{-3}$ withstand temperatures down to $-196^{\circ} \mathrm{C}$. Mostly for this reason, as compared to other insulating materials, a polyurethane rigid foam is highly competitive (Demharter, 1998). Beyond its low thermal conductivity, polyurethane has high mechanical properties at low temperature (Stirna et al., 2011). This is a key factor as the material remains stable despite the rising pressure of nitrogen vapors. The thermal insulating coverage was on purpose fabricated and fitted on the basis of the cryo-cabin dimensions: it was designed with a circular shape, with a maximum diameter of $102 \mathrm{~cm}$ and a minimum diameter of $93 \mathrm{~cm}$. A $21-\mathrm{cm}$-diameter hole was created in 
the middle of the layer to permit the head to remain outside the cabin. The coverage was made with a thickness of $5 \mathrm{~cm}$, using a polyurethane with a density of $180 \mathrm{~kg} \mathrm{~m}^{-3}$.

\subsection{STATISTICAL ANALYSIS}

All data are presented as mean \pm standard deviation. Gaussian distribution of the data was checked by skewness and kurtosis indexes both within the conventional cut-off (Groeneveld and Meeden, 1984).

Mixed model analyses of variance were applied to body surfaces' temperature, inner temperature of the cryo-cabin, and thermal discomfort. This approach was chosen to take into account the intrinsic (and uncontrolled) variability among the participants, which was considered everywhere as a random factor. Then, according to the aimpoints, a different analysis was conducted:

- As to body surfaces' temperature: a mixed model analysis of variance was applied to evaluate the effect of coverage ( 2 levels: open, covered) separately for each body regions (16 body surfaces, 8 front, and 8 rear). Data were introduced as mean of the five timepoints, since the main aim was to compare the two coverage conditions and because differences among the evaluation period were evaluated in a different analysis (see afterwards).

- Inner temperature of the cryo-cabin was examined by running two mixed model analyses of variance, one for each position - front $(n=10)$ and back $(n=9)$ - with coverage $(2$ levels: open, covered). Data were introduced as mean of the eleven timepoints for the same reason explained before. The dependent variable was still temperature.

- Participants' thermal discomfort (mean data over 9 timepoints) perceived through differentiated PBC session was analyzed with a mixed model analysis of variance to estimate the effect of coverage (2 levels: open, covered). 
According to the trapezoidal rule, the area under the curve (AUC) was used as a summary measure of cryo-cabin temperature registered during the cryostimulation session (every $15 \mathrm{~s}$, from 0 to $150 \mathrm{~s}=11$ timepoints). Likewise, the AUC of the thermal discomfort described the variation of the thermal discomfort perceived as function of time (from 30 to $150 \mathrm{~s}$, every 15 $\mathrm{s},=9$ timepoints). Comparisons among means of the AUCs were performed by using twotailed paired t-test.

Partial eta squared $\left(\eta_{\mathrm{p}}^{2}\right)$ effect sizes (ES) were determined and interpreted using the following cutoffs: small effect, $\eta_{\mathrm{p}}{ }^{2} \leq 0.03$; medium/moderate effect, $0.03<\eta_{\mathrm{p}}{ }^{2}<0.10$; large effect, $0.10 \leq \eta_{\mathrm{p}}^{2}<0.20$; very large effect, $\eta_{\mathrm{p}}^{2} \geq 0.20$ (Cohen, 1988).

Analyses were carried out with the Statistical Package SPSS version 26 for Mac (Armonk, NY, USA; IBM Corp.) and GraphPad Prism 9 (San Diego, CA, USA).

For all analyses, a p-value less than 0.05 was considered statistically significant.

\section{RESULTS}

\subsection{BODY SURFACES PROFILES OF THE TEMPERATURE: THERMAL IMAGES}

\section{OUTPUTS}

A significant effect of coverage appeared in chest-back $\left(F_{(1,17)}=20.40, P=0.0003, \eta_{p}^{2}=\right.$ $0.545)$, right forearm back $\left(\mathrm{F}_{(1,17)}=7.166, \mathrm{P}=0.016, \eta_{\mathrm{p}}{ }^{2}=0.297\right)$ and left forearm back $\left(\mathrm{F}_{(1,}\right.$ 17) $\left.=22.879, \mathrm{P}=0.0002, \mathrm{\eta}_{\mathrm{p}}{ }^{2}=0.574\right)$. In all those regions the temperature was significantly higher in open than in covered condition (chest back: open $27.61 \pm 1.24^{\circ}$, covered $26.29 \pm 0.81^{\circ}$; right forearm back: open $27.72 \pm 3.27^{\circ}$, covered $27.08 \pm 3.49^{\circ}$; left forearm back: open $21.77 \pm 1.65^{\circ}$, covered $20.20 \pm 1.79^{\circ}$ ). No significant effect of coverage was found in the 
temperature of the other body regions. A graphical representation of the results related to each timepoint and each body region is offered in Figure 4.

\subsection{INNER TEMPERATURE OF THE CRYO-CABIN}

In front position, the statistical analysis showed significant effects of coverage $\left(\mathrm{F}_{(1,9)}=\right.$ $\left.12.296, \mathrm{P}=0.007, \eta_{\mathrm{p}}^{2}=0.59\right)$ since front inner cryocabin temperature was lower in covered $(-$ $\left.59.58 \pm 1.04^{\circ}\right)$ that in open $\left(-54.74 \pm 1.73^{\circ}\right)$ condition. In back position no significant effect of coverage appeared $\left(\mathrm{F}_{(1,8)}=12.121, \mathrm{P}=0.833, \mathrm{\eta}_{\mathrm{p}}{ }^{2}=0.047\right)$.

The AUC_FRONT of the temperature*time (Fig. 5C) was greater in the covered condition $(\mathrm{t}(9)=$ 3.394, $\mathrm{P}=0.003)$, in absolute values, as compared to open condition $(-6,476 \pm 491.6$ vs $\left.5,769 \pm 751.3{ }^{\circ} \mathrm{C} \cdot \min \right)$, confirming a colder timecourse in the the covered cryo-cabin. The AUC_васк (Fig. 5D) showed no differences between the two conditions $(\mathrm{t}(0)=0.514, \mathrm{P}=$ 0.62; open: $-4,124 \pm 1038{ }^{\circ} \mathrm{C} \cdot \min$; covered: $\left.-4010 \pm 720{ }^{\circ} \mathrm{C} \cdot \min \right)$. The mean AUC relative values are represented in Figure 5, under different conditions.

\subsection{THERMAL DISCOMFORT}

The statistical analysis on the mean thermal discomfort over time did not find any significant effect of coverage $\left(\mathrm{F}_{(1,17)}=0.068, \mathrm{P}=0.798, \mathrm{\eta}_{\mathrm{p}}{ }^{2}=0.004\right)$. However, the AUC confirmed an effect of time with a greater thermal discomfort for the "covered" condition $(t(17)=3.397, \mathrm{P}$ $=0.003)$ compared to the open cryo-cabin session $(750.6 \pm 175.8$ vs $621.9 \pm 140.7 \mathrm{~cm} \cdot \mathrm{min})$. Graphical representation of AUC deploys the corresponding mean values in Figure 6.

\section{DISCUSSION}


The aim of this study was to compare thermal responses after a standardized protocol of PBC in an open cryo-cabin and following a PBC session into a cryo-cabin closed at the top with a polyurethane-made lid. Our hypothesis was confirmed as skin temperature of the upper body, in the front region, was significantly lower after exposure in a cryo-cabin covered with a polyurethane-made lid. For the first time, the use of a coverage placed on the top of a cryocabin was demonstrated to be thermo-effective and safe for PBC-practitioners and clinicians. Intriguingly, in recent years, some cryogenic equipment manufacturers have launched cryocabins with a standard coverage with the aim of lowering the actual procedure temperature. At the same time, there has been an increasingly diffusion of images of covered cryo-cabins, both on the social media and on the webpages of some cryotherapy facilities. Occasionally these pieces of equipment were even reported as self-produced. Despite of this new tendence, to our best knowledge, there are no studies that have investigated the thermo-effectiveness of this coverage. It is worth noting that, currently, standardized methods to assess inner temperature of these cryo-devices are deceiving. In fact, with rare exceptions, the site of measurement of inner temperature inside these technologies remains unknown (Bouzigon et al, 2016). Savic et al. (Savic et al., 2013) has documented an inner temperature of the cryocabin similar to that one we have utilized in this design. Moreover, inner cabin temperature next to the skin was greater than that one shown on the display of the cabin. Additionally, skin temperature was monitored up to 60 min after the cryostimulation session. The authors reported large epidermic temperature variations across body regions. The skin temperature had a significant drop after PBC, and skin temperature reflected this drop among different positions. However, in that study the inner cabin temperature was assessed by means of a manikin. In our study we tested the actual temperature inside the cabin with healthy male subjects both with a standard condition (Fonda et al., 2014) and with a coverage at the top of the cryo-cabin. In accordance with Savic and colleagues (Savic et al., 2013), we found a not 
uniform internal temperature, with a colder exposure in the front and a main effect of coverage. In detail, there was a mismatch between the temperature displayed at the machine (measured at the outlet of the nitrogen nozzle as shown in Fig 2) and the actual one - which was higher - measured at the front and the back of the subject (Fig. 5). Finally, in line with with Savic et al. (Savic et al., 2013), skin temperature had a significant drop after PBC, and those deltas of temperature varied to a different extent according to the positions of the thermocouple or the body regions (Fig.4). These study findings confirm and extend those of Savic, showing that the utilization of an insulated coverage led to obtain a lower inner temperature of the cabin (Fig. 5) and reduced temperatures for most of body surfaces with respect to control condition (Fig. 4). Therefore, this study addresses a critical issue regarding the efficiency of PBC devices in measuring the variation of the individual's skin temperature before and after cryo-exposure (Hausswirth et al., 2013). Most notably, this study provides additional evidence on the effectiveness of a polyurethane coverage at the top of a cryo-cabin for preserving lower inner cabin temperature as well as lower skin temperature. Another couple of complementary observations can be further discussed.

First, the polyurethane insulate coverage resulted to be a relatively uncostly solution, technically operable, and validated: these data attested the cryogenic effectiveness both inside the cabin and at epidermic site, therefore improving the overall efficiency of each cryosession. Second, it is known that PBC technology is based on direct contact between the subject and nitrogen gases. The subject's head must be out of the cabin because free nitrogen vapors in a cryo-cabin could be potentially hazardous due to the risk of asphyxia (EIGA, 2018), which is an important safety problem. The utilization of a coverage impedes the nitrogen gases to reach the head region, ensuring an optimal level of safety for the PBC procedure. 
Nevertheless, some limitations are worth noting. Although representative of the inner sites of the cryo-cabin, the internal temperature of the cabin was thorough documented in two locations only (Fig. 2). This can represent a weakness, as the analysis verified a not-uniform thermal distribution.

In second place, the study sample was composed by male participants only. A mono-gender dataset may lack generalizability of the results. In fact, it is known that the duration of cryostimulation protocols, under standard conditions, should be different when considering female compared to male subjects (Polidori et al., 2018). Future work should thus include both genders, various cryo-cabins and different time of exposure. This should permit to achieve a comprehensive spectrum of options, offering the best suited protocol for each desired coverage scenario.

In conclusion, practitioners and clinicians making use of PBC can successfully cover cryocabins with an insulating lid so to maintain lower skin temperature of the users, improving the effectiveness of the treatment. This procedure met the safety standards. Even perceived thermal discomfort implied that using a cover would not bear negative effects on the subjects' well-being. 


\section{Figure Captions}

Figure 1. Examples of thermal images taken before (A, front; B, back) and immediately after the PBC session ( $\mathrm{C}$, front; $\mathrm{D}$, back).

Figure 2. Schematic cross section of the cryo-cabin. The Thermocouple thermometer was placed nearby the shank (front) and the calf (back) of the subject.

Figure 3. Vertical section photo of the polyurethane coverage mounted at the top the cryocabin. In the middle, a $21-\mathrm{cm}-\varnothing$ hole is appreciable.

Figure 4. Variation of the individuals' skin temperature before (pre) and after (1, 5, 10, 20 minutes) cryo-exposure, measured in the front (A, open; B, covered) and the back (C, open; $\mathrm{D}$, covered) of several body surfaces. Data are presented as mean.

Figure 5. Inner temperature of the cryo-cabin measured in the front (A) and the (back) under open (grey dot) and covered (white square) conditions. The areas under the curve are respectively shown in $\mathrm{C}$ (front) and $\mathrm{D}$ (back). Data are presented as mean $\pm \mathrm{SD}$.

Figure 6. A) Thermal discomfort (VAS) during the cryostimulation session under open (grey) and covered (white) conditions. B) The area under the curve of the thermal discomfort was greater during the "covered" condition compared to the open $\mathrm{PBC}$ session $(\mathrm{t}(17)=3.397, \mathrm{P}=$ 0.003). Data are presented as mean $\pm \mathrm{SD}$. 


\section{References}

Bouzigon, R., Arfaoui, A., Grappe, F., Ravier, G., Jarlot, B., Dugue, B., 2017. Validation of a new whole-body cryotherapy chamber based on forced convection. J. Therm. Biol. 65, 138-144. https://doi.org/10.1016/j.jtherbio.2017.02.019

Bouzigon, R., Grappe, F., Ravier, G., Dugue, B., 2016. Whole- and partial-body cryostimulation/cryotherapy: Current technologies and practical applications. J. Therm. Biol. https://doi.org/10.1016/j.jtherbio.2016.08.009

Bouzigon, R., Ravier, G., Dugue, B., Grappe, F., 2014. THE USE OF WHOLE-BODY CRYOSTIMULATION TO IMPROVE THE QUALITY OF SLEEP IN ATHLETES DURING HIGH LEVEL STANDARD COMPETITIONS. Br. J. Sports Med. 48, 572.1572. https://doi.org/10.1136/bjsports-2014-093494.33

Cohen, J., 1988. Statistical power analysis for the behavioral sciences (revised ed.) Academic Press. New York. https://doi.org/10.1234/12345678

Costello, J.T., Culligan, K., Selfe, J., Donnelly, A.E., 2012. Muscle, skin and core temperature after $-110^{\circ} \mathrm{c}$ cold air and $8^{\circ} \mathrm{c}$ water treatment. PLoS One $7,1-8$. https://doi.org/10.1371/journal.pone.0048190

De Nardi, M., Facheris, C., Ruggeri, P., La Torre, A., Codella, R., 2020. High-impact Routines to Ameliorate Trunk and Lower Limbs Flexibility in Women. Int. J. Sports Med. https://doi.org/10.1055/a-1119-7902

De Nardi, M., La Torre, A., Benis, R., Sarabon, N., Fonda, B., 2015. Acute effects of wholebody cryotherapy on sit-and-reach amplitude in women and men. Cryobiology 71, 511513. https://doi.org/10.1016/j.cryobiol.2015.10.148

De Nardi, M., Pizzigalli, L., Benis, R., Caffaro, F., Cremasco, M.M., 2017. Acute effects of partial-body cryotherapy on isometric strength: Maximum handgrip strength evaluation. J. Strength Cond. Res. 31, 3497-3502. https://doi.org/10.1519/JSC.0000000000001797

Demharter, A., 1998. Polyurethane rigid foam, a proven thermal insulating material for applications between $+130^{\circ} \mathrm{C}$ and $-196^{\circ} \mathrm{C}$. Cryogenics (Guildf). 38, 113-117. https://doi.org/10.1016/S0011-2275(97)00120-3

Douzi, W., Dupuy, O., Theurot, D., Boucard, G., Dugué, B., 2019. Partial-body cryostimulation after training improves sleep quality in professional soccer players. BMC Res. Notes 12. https://doi.org/10.1186/s13104-019-4172-9

Ferreira-Junior, J.B., Bottaro, M., Vieira, A., Siqueira, A.F., Vieira, C.A., Durigan, J.L.Q., Cadore, E.L., Coelho, L.G.M., Simões, H.G., Bemben, M.G., 2015. One session of partial-body cryotherapy $\left(-110^{\circ} \mathrm{C}\right)$ improves muscle damage recovery. Scand. J. Med. Sci. Sport. 25, e524-e530. https://doi.org/10.1111/sms.12353

Fonda, B., De Nardi, M., Sarabon, N., 2014. Effects of whole-body cryotherapy duration on thermal and cardio-vascular response. J. Therm. Biol. 42, 52-55. https://doi.org/10.1016/j.jtherbio.2014.04.001

Hausswirth, C., Schaal, K., Le Meur, Y., Bieuzen, F., Filliard, J.-R., Volondat, M., Louis, J., 2013. Parasympathetic Activity and Blood Catecholamine Responses Following a Single Partial-Body Cryostimulation and a Whole-Body Cryostimulation. PLoS One 8, e72658. https://doi.org/10.1371/journal.pone.0072658

Ksiezopolska-Pietrzak, K., 2000. Cryotherapy in the treatment of rheumatic disease. Ortop. Traumatol. Rehabil. 2.

Leon, G.R., Koscheyev, V.S., Stone, E.A., 2008. Visual Analog Scales for Assessment of Thermal Perception in Different Environments. Aviat. Space. Environ. Med. 79, 784 786. https://doi.org/10.3357/ASEM.2204.2008 
Louis, J., Schaal, K., Bieuzen, F., Le Meur, Y., Filliard, J.R., Volondat, M., Brisswalter, J., Hausswirth, C., 2015. Head exposure to cold during whole-body cryostimulation: Influence on thermal response and autonomic modulation. PLoS One 10, 1-18. https://doi.org/10.1371/journal.pone.0124776

Matos, F., Neves, E.B., Norte, M., Rosa, C., Reis, V.M.H., Vilaça-Alves, J., 2015. The use of thermal imaging to monitoring skin temperature during cryotherapy: A systematic review. Infrared Phys. Technol. 73, 194-203. https://doi.org/10.1016/j.infrared.2015.09.013

Piras, A., Cortesi, M., Campa, F., Perazzolo, M., Gatta, G., 2019. Recovery time profiling after short-, middle-and long-distance swimming performance. J. Strength Cond. Res. 33. https://doi.org/10.1519/jsc.0000000000002066

Polidori, G., Taiar, R., Legrand, F., Beaumont, F., Murer, S., Bogard, F., Boyer, F.C., 2018. Infrared thermography for assessing skin temperature differences between Partial Body Cryotherapy and Whole Body Cryotherapy devices at $-140^{\circ} \mathrm{C}$. Infrared Phys. Technol. 93, 158-161. https://doi.org/10.1016/j.infrared.2018.07.025

Ring, E.F.J., Ammer, K., 2015. The technique of infrared imaging in medicine. Infrared Imaging A Caseb. Clin. Med. 7-14. https://doi.org/10.1088/978-0-7503-1143-4ch1

Ring, E.F.J., Ammer, K., 2012. Infrared thermal imaging in medicine. Physiol. Meas. 33. https://doi.org/10.1088/0967-3334/33/3/R33

Rivera, J., Tercero, M.J., Salas, J.S., Gimeno, J.H., Alejo, J.S., 2018. The effect of cryotherapy on fibromyalgia: a randomised clinical trial carried out in a cryosauna cabin. Rheumatol. Int. 38, 2243-2250. https://doi.org/10.1007/s00296-018-4176-0

Savic, M., Fonda, B., Sarabon, N., 2013. Actual temperature during and thermal response after whole-body cryotherapy in cryo-cabin. J. Therm. Biol. 38, 186-191. https://doi.org/10.1016/j.jtherbio.2013.02.004

Stirna, U., Beverte, I., Yakushin, V., Cabulis, U., 2011. Mechanical properties of rigid polyurethane foams at room and cryogenic temperatures. J. Cell. Plast. 47, 337-355. https://doi.org/10.1177/0021955X11398381

YAMAUCHI, T., 1986. Whole Body Cryotherapy is Method of extreme Cold $-175^{\circ} \mathrm{C}$ Treatment initially used for Rheumatoid Arthritis. Z. Phys. Med. Baln. Med. Klim 15, 311.

Zagrobelny, Z., Halawa, B., Negrusz-Kawecka, M., Spring, A., Gregorowicz, H., Wawrowska, A., Rozwadowski, G., 1992. Hormonal and hemodynamic changes caused by whole body cooling in patients with rheumatoid arthritis. Pol. Arch. Med. wewntrznej 87. 